\title{
New Insights into the Special Theory of Relativity
}

\author{
Amrit S. Sorli, ${ }^{\text {a) }}$ Dusan Klinar $^{\text {b) }}$ and Davide Fiscaletti ${ }^{\text {c) }}$ \\ Scientific Research Centre Bistra, Slovenski trg 6, 2250 Ptuj, Slovenia \\ Received 24 February 2011, accepted 20 April 2011 Physics Essays - AIP, Vol.24, Num.2, 2011 \\ http://www.physicsessays.com/
}

\begin{abstract}
In the $20^{\text {th }}$ century, physics has understood space and time as being coupled into a "spacetime" manifold, a fundamental arena in which everything takes place. Space-time was considered to have three spatial dimensions and one temporal dimension. Out of the mathematical formalism for the fourth space-time component $\mathrm{X} 4=$ ict one can conclude that time $\mathrm{t}$ is only a numerical order of material change, i.e., the motion that we obtain with clocks. Time is not a $4^{\text {th }}$ dimension of space. For the description of the Special Theory of Relativity it is here proposed an Minkowski 4D space whilst time $t$ is merely a numerical order of a photon motion in a 4D space. This view opens new perspectives on the understanding of the quantum entanglement, where the 4D space becomes an immediate medium for quantum communication.
\end{abstract}

Key words: space-time, space, time, numerical order, time dilation, light clock, quantum entanglement.

\section{According to the mathematical formalism $X_{4}=i c t$ in the Special Theory of Relativity time $t$ is a numerical order of material changes that run in a 4D space}

According to the mathematical formalism of $X_{4}=i c t$ the space-time is a four dimensional space with its dimensional components $X_{1}, X_{2}, X_{3}, X_{4}$ where the fourth coordinate is $X_{4}=i c t$. $X_{4}$ is formulated as a product of an imaginary number $i(i=\sqrt{-1})$, light speed $c$, and time $t$. In this formula, the time $t$ represents the numerical order of a physical event which is measured with clocks. Comparing the formula $X_{4}=i c t$ with the fundamental physical formula which expresses the relation between distance $d$, velocity $v$, and time $t$ namely $d=v t$ we see immediately that $X_{4}$ is a spatial distance. Considering time as being the fourth dimension of space does not seem appropriate from the physical point of view. It is more appropriate to see the Minkowskian arena as a 4D space rather than a $3 D+T$ space-time. Some relevant theoretical results (regarding state space, for example) and experimental results (regarding the "immediate physical phenomena", namely phenomena that occur with an elapsed clock run equal to zero) suggest that the standard space-time cannot be considered as the fundamental arena of the universe and thus that the space-time manifold characteristic of the standard interpretation of special relativity must be replaced with a 4D space where time exists only as a measuring system of the numerical order of material change.

a) sorli@spacelife.si

b) dusan@bistra.si

c) fiscalettidavide@libero.it 
According to several current studies, the ordinary space-time does not correspond indeed to a physical reality and a "state space" can be proposed as the fundamental arena. For example, Girelli, Liberati and Sindoni have recently developed a toy model in which they have shown how the Lorentzian signature and a dynamical space-time can emerge from a non-dynamical Euclidean space, with no diffeomorphisms invariance built in. In this sense this toy-model provides an example where time (from the geometric perspective) is not fundamental, but simply an emerging feature. ${ }^{1}$ In more detail, this model suggests that at the basis of the arena of the universe there is some type of "condensation", so that the condensate is described by a manifold $R^{4}$ equipped with the Euclidean metric $\delta^{\mu v}$. Both the condensate and the fundamental theory are timeless. The condensate is characterized by a set of scalar fields $\Psi_{i}\left(x_{\mu}\right), \mathrm{i}=1,2,3$. Their emerging Lagrangian $\mathrm{L}$ is invariant under the Euclidean Poincarè group $\mathrm{ISO}(4)$ and has thus the general shape

$$
L=f\left(X_{1}\right)+f\left(X_{2}\right)+f\left(X_{3}\right) ; X_{i}=\delta^{\mu v} \partial_{\mu} \Psi_{i} \partial_{v} \Psi_{i}
$$

The equations of motion for the fields $\Psi_{i}\left(x_{\mu}\right)$ are simply given by

$$
\partial_{\mu}\left(\frac{\partial L}{\partial X_{i}} \partial^{\mu} \Psi_{i}\right)=0=\sum_{j}\left(\frac{\partial^{2} L}{\partial X_{i} \partial X_{j}}\left(\partial^{\mu} X_{j}\right)+\frac{\partial L}{\partial X_{i}} \partial_{\mu} \partial^{\mu} \Psi_{i}\right)
$$

The fields $\Psi_{i}\left(x_{\mu}\right)$ can be expressed as $\Psi_{i}=\psi_{i}+\varphi_{i}$ where $\varphi_{i}$ are the perturbations around the solutions $\psi_{i}$ of the above equation.

Different choices of the solutions $\psi_{i}$ lead to different metrics

$$
g_{k}^{\mu \nu}=\frac{d f}{d X_{k}}\left(\bar{X}_{k}\right) \delta^{\mu \nu}+\frac{1}{2} \frac{d^{2} f}{\left(d X_{k}\right)^{2}}\left(X_{k}\right) \partial^{\mu} \psi_{k} \partial^{v} \psi_{k} \text { (3). }
$$

and the Lorentzian signature and the Minkowski metric can be obtained for the condition $\frac{d f}{d X}(\bar{X})+\frac{\alpha^{2}}{2} \frac{d^{2} f}{(d X)^{2}}(\bar{X})<0, \frac{d f}{d X}(\bar{X})>0$.

The toy model developed by Girelli, Liberati and Sindoni shows in a clear way that at a fundamental level the background space of physical events is a timeless condensate and that different solutions of the equations of motion of the fields characterizing this condensate determine different metrics of the space-time background. This means that on the basis of this model time as humans perceive it cannot be considered a fundamental physical reality, the duration of material change has no physical existence of its own. On the basis of the model of Girelli, Liberati and Sindoni, the suggestive idea can be proposed that the arena of the universe is a 4D space where clock/time is only a mathematical coordinate, is only a measuring device for motion and thus that the Minkovskian arena of special relativity must be interpreted as a 4D space. In the Special Relativity time $t$ is not a dimension of space, time t merely is the numerical order of change in space we measure with clocks.

The Planck's time is a fundamental unit of the numerical order of physical events. Let us consider light. A photon moves in space. Let us observe a photon moving from point A to point B in space. By assuming that space has a granular structure at the Planck scale (this view is supported by significant theoretical results regarding, for example, reticular space-time dynamics and loop quantum gravity ${ }^{2,3,4}$ ), the smallest distance that a photon can move on the distance $d_{A B}$ from $\mathrm{A}$ to $\mathrm{B}$ is the Planck's length 
$l_{p}=\sqrt{\frac{\hbar G}{c^{3}}}: d_{A B}=l_{p 1}+l_{p 2} \ldots+l_{p n}$. Each motion on the line segment $l_{p x}$ corresponds exactly to one Planck time $t_{p x}=\sqrt{\frac{\hbar G}{c^{5}}}$. The time $t$ of photon motion across distance $d_{A B}$ is the sum of all $t_{p x}$

$$
t=t_{p 1}+t_{p 2} \ldots+t_{p n}=\sum_{x=1}^{n} t_{p x}
$$

The Planck's time $t_{p}$ is here considered to be the fundamental unit for measuring the numerical order of photon motion. The velocity of a photon $c$ is calculated by dividing the Planck's length $l_{p}$ with Planck's time $t_{p}$. For longer distances $d_{A B}, c$ is obtained by dividing $d_{A B}$ with the numerical order of the photon motion $t$ :

$$
c=\frac{l_{p}}{t_{p}}=\frac{d_{A B}}{t}
$$

The symbol of time $t$ in all mathematical formalisms of physics is a number which represents the numerical order of material changes, i.e. motion. ${ }^{5,6}$

\section{Historical overview of space-time as the fundamental arena of the universe}

Let us see how the idea of "space-time" as the fundamental arena of the universe has entered into physics. Experiments with light carried out in the last decades of the $19^{\text {th }}$ century confirmed that, for an observer, light has the same speed regardless of whether the source of light is moving towards the observer or is moving away from the observer. The Galilean transformation which has served physicists for centuries to describe the positions of two inertial systems proved unfit to describe this unusual property of light. Let us suppose that one observer is standing at a train station (observer $\mathrm{O}$ ), and another observer is on the train itself (observer O'). At the moment the train passes the station, we start measuring the numerical order of the train's motion with a clock. At any distance from the station we can describe the position between these two observers in terms of Galilean transformation.

In Galilean transformation the clock at the train-station and the clock on the train run with the same rate: $t^{\prime}=t$. This works fine when the velocity of the train is low, i.e., between 100 and $200 \mathrm{~km} / \mathrm{h}$. If we imagine a train with a speed close to that of an airplane or higher, we can no more describe the position of observers $\mathrm{O}$ and $\mathrm{O}^{\prime}$ ' with a Galilean transformation because light still has the same speed for both observers. To solve this puzzle of the constancy of the speed of light, Einstein in his Special Theory of Relativity used the Lorentz transformation which exactly describes the position of observers $\mathrm{O}$ and $\mathrm{O}^{\prime}$, also when $O^{\prime}$ on the train moves with a speed $v$ close to the light speed $c$. According to the Lorentz transformation, the clock on the super fast train runs slower than the clock at the train station. The higher the speed of the train, the slower the speed of the clock on it in comparison with the speed of the clock at the train-station. These different clock speeds have been proved experimentally, clocks actually run slower on a fast airplane than on the surface of the Earth. 
Let us take a look at a classical example: the train passes the train station with a velocity of $1,000.00 \mathrm{~km} / \mathrm{h}$, i.e. $277.78 \mathrm{~m} / \mathrm{s}$. Both clocks start running at the exact moment when the compartment with the clock passes the train station. After exactly one hour the train stops at the next train station. The clock o (station clock) is programmed to stop exactly after $3,600.00$ seconds, the clock o' (train clock) is programmed to stop exactly after moving on a distance $d=1000,000.00 \mathrm{~m}$. The distance $d$ has the same length for both observers $\mathrm{O}$ and $\mathrm{O}^{\prime}$. When the train has stopped at the train station and clocks have stopped, observers $\mathrm{O}$ and $\mathrm{O}^{\prime}$ use their mobile phones and inform each other about the clocks' rate.

Calculations according to the Lorentz formula of the transformation of the speed of clocks $t^{\prime}=\frac{t-\frac{v X}{c^{2}}}{\sqrt{1-\frac{v^{2}}{c^{2}}}}$

(or, as the reader can easily check, according to the equivalent formula $t^{\prime}=t * \sqrt{1-\frac{v^{2}}{c^{2}}}$ that we will see in detail in chapter IV) show that, for both of observers, the clock o (station clock) indicates 3,600.00 seconds, and the clock o' (train clock) indicates 3,599.99999999845 seconds. The clock o' "ticks" slower by 0.00000000155 seconds. This difference in clock speeds is true for both observers. In inertial systems $\mathrm{O}$ and $\mathrm{O}$ ' there is no "proper time" in which clocks run. The train moves and clocks run in space only, not in time. In inertial systems, there are only "proper velocities of clocks" which depend on the velocity of inertial systems themselves. These different clock speeds in different inertial systems have been proved experimentally, clocks actually run slower on a fast airplane than on the surface of the Earth.

\section{The common interpretation of how an observer at rest experiences the velocity of a "light clock" in a moving inertial system seems not adequate}

Fig. 1 shows a common interpretation of "light clocks" in Special Relativity from the point of view of the observer $O$ who is at rest. The clock $O$ on the left is at rest, the clock o' on the right is moving with constant velocity in horizontal direction. According to the common interpretation for the observer $\mathrm{O}$ at the station, the moving clock o' is supposed to run ("tick") slower than the clock at rest o because the path of the photon seems longer than by the clock at rest. One "tick" of the clock means a photon passes one distance between the two mirrors. ${ }^{7}$

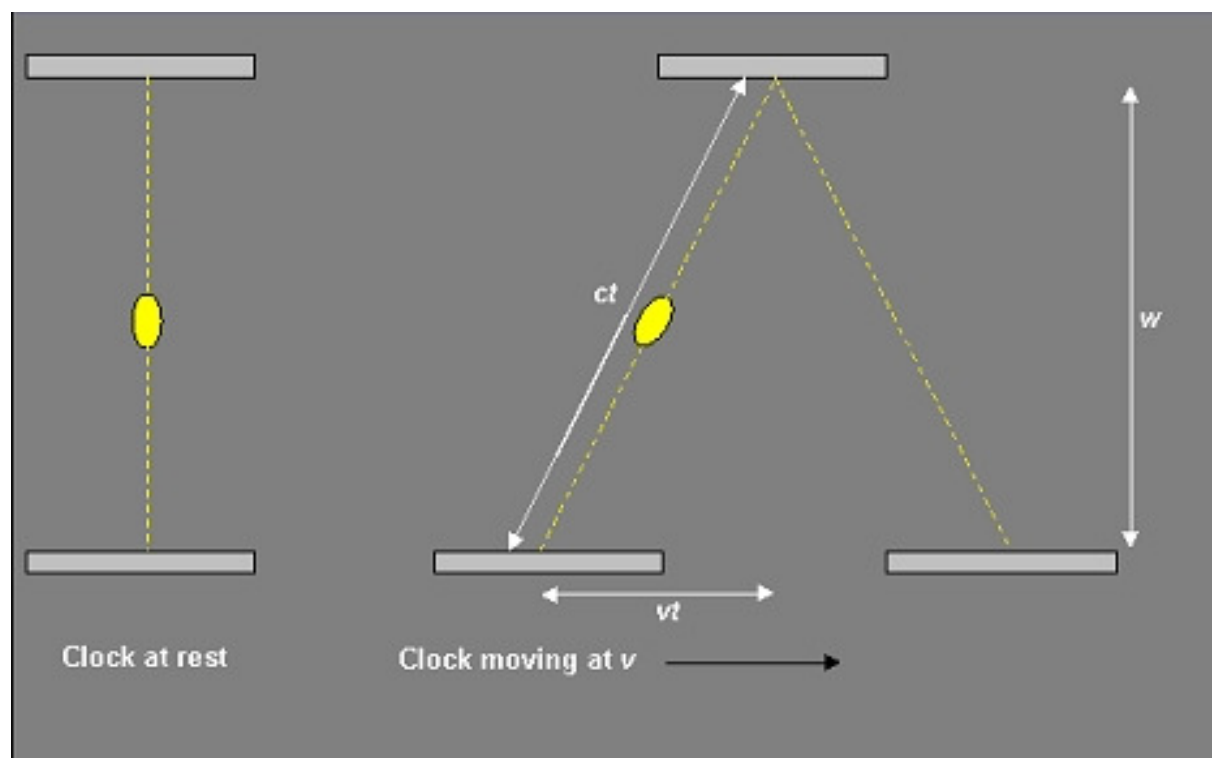


FIG. 1. Two identical light clocks: one at rest, one moving relative to us. The light blips in both travel at the same speed relative to us, the one in the moving clock goes further, so must take longer between clicks. $t$ is the time from one mirror to the other.

The interpretation of the authors of this article is the following: the observer $O$ experiences that photon of the moving clock o' has a longer path between mirrors only because the clock o' is moving. This 'illusive experience" caused by motion does not mean that for the observer $O$ the moving clock 0 ' will have a slower rate. In the moving clock o' photon moves in a vertical direction and has the same path between mirrors as in the clock $o$ at rest. When we spill water from a glass on board of a flying airplane, water will fall down vertically from the glass. If a ball is thrown perpendicularly towards the floor in a flying airplane, it will bounce back from the floor vertically. Take a sand clock in an airplane. By assuming that air does not provide resistance, the sand will fall down vertically as if it were on the surface of the Earth. Also a photon in a moving clock o' moves in a vertical direction. Out of this the following consequences can be drawn:

a) The photons in a clock at rest $\mathrm{o}$ and in a moving clock o' move in a vertical direction.

b) The path of the photons between mirrors is equal in both clocks 0 and o'.

c) The velocity $c$ of photons is the same in both clocks $o$ and o'.

The points a), b), c) are valid for both observers $O$ and $O$ '. This "thought experiment" with clock at rest $O$ and moving clock 0 ' seems to suggest that at the photon scale the "relative velocity" of the physical phenomena in different inertial systems is not valid any more. The "relative velocity" of material changes starts with the massive particles as it is proved by different decay time of rest pi mesons and pi mesons in motion and by different lifetimes of rest muons and muons in motion. ${ }^{8}$

\section{Lorentz transformations between spatial coordinates $X_{4}$ and $X_{4}^{\prime}$ and the ticking of clocks o and o'}

Considering time $t$ as a numerical order of material change in a 4D space, the authors propose that in a frame of Lorentz formalism there is an additional transformation between spatial coordinates $X_{4}$ and $X_{4}{ }^{\prime}$ :

$$
\left\{\begin{array}{c}
X_{1}^{\prime}=\left(X_{1}-v t\right) * \sqrt{1-\frac{v^{2}}{c^{2}}} \\
X_{2}^{\prime}=X_{2} \\
X_{3}^{\prime}=X_{3} \\
X_{4}^{\prime}=X_{4} * \sqrt{1-\frac{v^{2}}{c^{2}}} \\
t^{\prime}=t * \sqrt{1-\frac{v^{2}}{c^{2}}}
\end{array}\right.
$$

Existing formalisms of the Special Theory of Relativity $X_{4}=i * c * t, X_{4}{ }^{\prime}=i * c * t^{\prime}$ show that time $t$ is not the $4^{\text {th }}$ dimension of a four-dimensional Minkowski space: $X_{4} \neq t, X_{4}^{\prime} \neq t^{\prime}$. According to these formalisms it is not correct to consider time $t$ as being the fourth dimension $X_{4}$. The relation between the 
numerical order of clock's run $t$ and $t$ in a 4D Minkowski space has originally been expressed by the Lorentz formalism:

$$
t^{\prime}=\frac{t-\frac{v X}{c^{2}}}{\sqrt{1-\frac{v^{2}}{c^{2}}}}
$$

In order to develop a mathematical formalism which confirms that time $t$ is a distinct entity from space, let us make some manipulations inside Eq. (7). If we divide this equation by $t$, we obtain

$$
\frac{t^{\prime}}{t}=\frac{1-\frac{v X}{c^{2} t}}{\sqrt{1-\frac{v^{2}}{c^{2}}}}
$$

Now, on the right side $X$ divided by $t$ is just the velocity parameter $v$ if the motion happens with constant velocity. In this way we obtain

$$
\frac{t^{\prime}}{t}=\frac{1-\frac{v^{2}}{c^{2}}}{\sqrt{1-\frac{v^{2}}{c^{2}}}} \quad \text { namely } \quad \frac{t^{\prime}}{t}=\sqrt{1-\frac{v^{2}}{c^{2}}}
$$

By following this procedure, one arrives therefore at a time transformation of the form:

$$
t^{\prime}=\sqrt{1-\frac{v^{2}}{c^{2}}} * t \quad(8)
$$

i.e., that shows no space-dependence.

Out of Eq. (8) it follows:

$$
X^{\prime}{ }_{4}=i * c * t * \sqrt{1-\frac{v^{2}}{c^{2}}}=X_{4} * \sqrt{1-\frac{v^{2}}{c^{2}}}
$$

namely the fourth of Eqs. (6).

It is important to underline that a formula like Eq. (8) regarding the transformations of time has been found for example by Selleri.

In this regard, Selleri has introduced general transformations of space and time between inertial reference frames that seem to indicate clearly that space and time must be considered as separate entities: in special relativity time must be separated from space. ${ }^{9,10,11}$ Given the inertial frames $S_{0}$ and S' endowed with Cartesian coordinates (where the origin of S', observed from $S_{0}$, is seen to move with velocity $v<c$ parallel to the $+x_{0}$ axis), by starting from the following two empirically based assumptions:

1) the two-way velocity of light is the same in all directions and in all inertial systems; 
2) clock retardation takes place with the usual velocity dependent factor when clocks move with respect to $S_{0}$,

Selleri has found the following transformations for the space and time variables from $S_{0}$ to $S^{\prime}$ (called also equivalent transformations):

$$
\left\{\begin{array}{c}
x^{\prime}=\frac{x_{0}-v t_{0}}{\sqrt{1-\beta^{2}}} \\
y^{\prime}=y_{0} \\
z^{\prime}=z_{0} \\
t^{\prime}=\sqrt{1-\beta^{2}} t_{0}+e_{1}\left(x_{0}-v t_{0}\right)
\end{array}\right.
$$

where $\beta=\frac{v}{c}$. These transformations contain a free parameter, $e_{1}$, the coefficient of $x$ ' in the transformations of time (namely of the speed of clocks) which can be fixed by choosing a clock synchronization method in $S^{\prime}$. Different values of $e_{1}$ are thus obtained from different synchronization conventions. The special theory of relativity (and thus the standard Lorentz transformations) is obtained for a particular choice of $e_{1}: e_{1}=-\frac{\beta}{c \sqrt{1-\beta^{2}}}$. For all the values of $e_{1}$ except the case of the special theory of relativity, the system $S_{0}$ turns out to be a privileged reference frame. As it has been analyzed by Selleri, different values of $e_{1}$ determine different theories of space and time which are empirically equivalent to a large extent. Michelson-type experiments ${ }^{12}$, the twin paradox experiment ${ }^{13}$, radar ranging of planets and occultation of Jupiter satellites ${ }^{14}$, Doppler effect and aberration ${ }^{15}$, Fizeau experiment ${ }^{16}$ were proved to be insensitive to the choice of $e_{1}$. These results show that Poincarè ${ }^{17}$, Reichenbach ${ }^{18}$, Jammer $^{19}$ and Mansour and Sexl ${ }^{20}$ were basically correct in stating that the clock synchronization in inertial systems is conventional and the assumed invariance of the one way velocity of light of the standard interpretation of the Special Theory of Relativity has only motivations of simplicity (No perfectly inertial reference frame exists in practice, e.g. because of Earth rotation around its axis, of orbital motion, etc... All we know about inertial systems has actually been obtained in reference frames endowed with a small but non zero acceleration. The frame associated with Earth for many problems can be considered inertial just because its rotation, being of a fourth order, can be neglected). But, as it has been shown by Selleri, there are some particular phenomena (the accelerating spaceships, the rotating disk and the question of superluminal signals regarding the group velocity of electromagnetic radiation) that modify the situation to the point that the condition $e_{1}=0$ becomes necessary. The adoption of $e_{1}=0$ makes Eqs. 9 become the "inertial transformations" in which the transformation of the speed of clock does not contain the space variable:

$$
\left\{\begin{array}{c}
x^{\prime}=\frac{x_{0}-v t_{0}}{\sqrt{1-\beta^{2}}} \\
y^{\prime}=y_{0} \\
z^{\prime}=z_{0} \\
t^{\prime}=\sqrt{1-\beta^{2}} t_{0}
\end{array}\right.
$$


The inverse transformations of these are:

$$
\left\{\begin{array}{c}
x_{0}=\sqrt{1-\beta^{2}}\left(x+\frac{v t^{\prime}}{1-\beta^{2}}\right) \\
y_{0}=y^{\prime} \\
z_{0}=z^{\prime} \\
t_{0}=\frac{t^{\prime}}{\sqrt{1-\beta^{2}}}
\end{array}\right.
$$

Equations Eqs. (10) and Eqs. (11) determine an arena of Special Relativity in which time $t$ must be clearly considered a different entity with respect to the spatial coordinates $x, y$ and $z$ just because the fourth of these equations indicates that the transformation of the speed of clocks between the two inertial systems does not depend on the spatial coordinates $x, y$ and $z$. In Eqs. (11) the transformation for the $4^{\text {th }}$ spatial

coordinate $X_{4}$ of Minkowski 4D space is missing; time $t$ is the numerical order of clock velocity and not a $4^{\text {th }}$ coordinate of $4 \mathrm{D}$ space. In Eqs. (6) this inaccuracy is improved.

With time $t$ as numerical order of change the "Twin paradox" is definitely resolved; the twin brother in a fast spaceship ages slower than his brother on Earth. Both are growing older in a space only where time $t$ is a numerical order of their aging. Also the question of time travels is definitely resolved: time travel into past or into future is not possible. One can travel in a space only and time $t$ is a numerical order of his/her motion.

\section{Quantum space is an immediate medium of quantum information transfer}

According to the concept of space-time, all physical phenomena happen in space and time. This concept cannot explain those physical phenomena where information transfer is immediate. For these phenomena time $t$ (i.e. the elapsed clock run for them to happen) is zero. If these phenomena would happen in time intended as some physical reality, time could never be zero. This article presents a new concept of time in quantum space where time $t$ is only a numerical order of material change: quantum phenomena where information transfer is immediate have no time. Examples of such phenomena are: the non-local correlations between quantum particles in EPR-type experiments and other immediate physical phenomena like tunneling or quantum entanglements regarding the continuous variable systems or the quantum excitations from one atom to another in Fermi's two-atom system. ${ }^{21,22,23,24}$ These phenomena are carried directly by the quanta of space QS which constitute quantum space. Quanta of space QS have a volume of Planck: $l_{p}^{3}=\sqrt{\frac{(\hbar G)^{3}}{c^{9}}}$, where $\hbar$ is the reduced Planck constant, $G$ is the gravitational constant and $c$ is the velocity of light. Inside Planck volume, time as numerical order of material change does not exist. Time enters existence at the scale of Planck. Planck time is the fundamental unit of photon motion on the Planck length. ${ }^{6}$ 


\section{Falsifiability of the here presented theory of time:}

Let us consider the falsifiability of the following two statements, A and B:

A. For all experiments, time $t$ is equally as space a fundamental physical entity in which a given experiment occurs;

B. For all experiments, time $t$, when measured with clocks, is merely a numerical order of material change taking place in space which is a fundamental physical entity in which a given experiment occurs.

Statement $A$ has no basis in the elementary visual perception. This is its weak point. Statement $A$ is falsifiable by an experiment in which time $t$ does not exist. Such an experiment is the Coulomb experiment with a torsion balance to measure electrostatic interaction between two metal-coated balls. The Coulomb experiment has the following mathematical formalism:

$$
F=k_{e} \frac{q_{1} * q_{2}}{r^{2}}(12)
$$

In this experiment, time is not present as the fundamental entity in which the experiment takes place. To consider statement $A$ as correct, it should be proven that the Coulomb experiment does not take place in space only but also in time, and that time does not affect the electrostatic interaction between metalcoated balls. Without this proof this experiment indicates statement A is wrong: formalism (12) indicates experiment takes place only in space as a fundamental physical entity, not in time.

In Newton's measuring of gravitational force between two material objects, time $t$ as the fundamental physical entity does not exist. In the formalism of gravitational force measurement between two material objects of masses $m_{1}$ and $m_{2}$ there is no time $t$ :

$$
F=\frac{m 1 * m 2 * G}{r^{2}}
$$

In this experiment, time is not present as the fundamental physical entity in which the experiment takes place. To consider statement $A$ as correct it should be proven that this experiment takes place in time and time does not affect gravitational force between two material objects. Without this proof the experiment indicates that statement $A$ is wrong: formalism (13) indicates the experiment takes place only in space as a fundamental entity, not in time.

Statement B has its basis in the elementary visual perception. This is its strong point. Ocular experience confirms that clocks measure the numerical order of material change in space as a fundamental entity in which an experiment occurs. Statement B is falsifiable by an experiment where time $t$ measured with clocks is not the numerical order of material change. Such an experiment would prove statement $B$ to be wrong; such an experiment is not known yet. An experiment where there is no time is not disproving statement B. 


\section{Conclusions}

In Minkowski space-time the fourth coordinate $X_{4}=i c t$ is not a temporal coordinate. Time $t$ as the numerical order of material change we measure with clocks is merely a component of a $X_{4}{ }_{4} X_{4}$ is spatial, too. Minkowski space is not $3 D+T$, it is $4 \mathrm{D}$. The point of view which considers time to be a physical entity in which material changes occur is here replaced with a more convenient view of time being merely the numerical order of material change. This view corresponds better to the physical world and has more explanatory power in describing immediate physical phenomena: gravity, electrostatic interaction, information transfer by EPR experiment are physical phenomena carried directly by the space in which physical phenomena occur.

\section{Acknowledgement:}

The authors wish to thank Erik Margan for his advices and encouragement.

${ }^{1}$ F. Girelli, S. Liberati, L. Sindoni, "Is the notion of time really fundamental?", submitted on 27 Mar 2009, available at http://arxiv.org/abs/0903.4876.

2 C. Rovelli, "Loop Quantum Gravity”, Living Reviews in Relativity, http://relativity.livingreviews.org/Articles//rr-1998-1/ (1997).

${ }^{3}$ C. Rovelli, Quantum Gravity (Cambridge University Press, Cambridge, 2004).

4 I. Licata, "Minkowski Space-Time and Dirac Vacuum as Ultrareferential Reference Frame", Hadronic Journal 14, 3 (1991).

${ }^{5}$ A. S. Sorli, D. Fiscaletti and D. Klinar, "Time is a measuring System derived from Light Speed", Physics Essays 23, 2, 330 (2010).

${ }^{6}$ A. S. Sorli, D. Fiscaletti and D. Klinar, "Replacing Time with Numerical Order of Material Change resolves Zeno Problems of Motion", Physics Essays 24, 1, 2011.

7 J. M. Levi, "The simplest derivation of Lorentz transformation", submitted on 12 Jun 2006, last revised 9 Oct 2006, http://arxiv.org/PS cache/physics/pdf/0606/0606103v4.pdf (2006).

8 G. Zanella, "Lorentz contraction or Lorentz dilation?", submitted on 28 Oct 2010, last revised 29 Oct 2010, arXiv:1010.5988v2 [physics.gen-ph].

${ }^{9} \mathrm{~F}$. Selleri (2000), "Space and time should be preferred to spacetime - 1", International workshop Physics for the $21^{\text {st }}$ century, 5-9 June 2000.

${ }^{10} \mathrm{~F}$. Selleri (2000), "Space and time should be preferred to spacetime - 2", International workshop Physics for the $21^{\text {st }}$ century, 5-9 June 2000.

${ }^{11}$ R. Manaresi and F. Selleri (2004), "The international atomic time and the velocity of light", Found. Phys. Lett. 17, 65. 
${ }^{12}$ F. Selleri, "Velocity-symmetrizing synchronization and conventional aspects of relativity", in Waves and particles in light and matter, pp. 439-446, A. van der Merwe et al. eds. (Plenum Press, London, 1994).

${ }^{13}$ F. Selleri, Found. Phys. Lett. 9, 43 (1996).

${ }^{14}$ F. Selleri, "Bell's spaceships and special relativity", in: R.A. Bertlmann and A. Zeilinger eds., Quantum unspeakables, from Bell to quantum information (Springer, Berlin, 2002).

${ }^{15}$ G. Puccini and F. Selleri, Nuovo Cimento B 117, 283 (2002).

${ }^{16}$ F. Selleri, Found. Phys. Lett. 16, 71 (2003).

${ }^{17}$ H. Poincarè, Rev. Metaphys. Morale 6, 1 (1898).

${ }^{18} \mathrm{H}$. Reichenbach, The philosophy of space and time (Dover, New York, 1958).

${ }^{19} \mathrm{M}$. Jammer, "Some fundamental problems in the special theory of relativity", in: G. Toraldo di Francia ed., Problems in the foundations of physics, pp. 202-236 (Società Italiana di Fisica, Bologna and North Holland, Amsterdam, 1979).

${ }^{20}$ R. Mansouri and R. Sexl, General Relat. Gravit. 8, pp. 497, 515, 809 (1977).

21 D. Fiscaletti and A.S. Sorli, "Non-locality and the Symmetryzed Quantum Potential", Physics Essays, 21, 4, 245 (2008).

${ }^{22}$ P. Eckle, A. N. Pfeiffer, C. Cirelli, A. Staudte, R. Dörner, H. G. Muller, M. Büttiker and U. Keller, "Attosecond lonization and Tunneling Delay Time Measurements in Helium", Science 322, 5907, 1525 1529 (2008), available at http://www.sciencemag.org/cgi/content/short/322/5907/1525.

23 G.C. Hegerfeldt. "Causality problems for Fermi's two-atom system”, Phys. Rev. Lett. 72, 596 - 599 (1994), available at http://prola.aps.org/abstract/PRL/v72/i5/p596 1.

24 T.N. Palmer, "The Invariant Set Hypothesis: A New Geometric Framework for the Foundations of Quantum Theory and the Role Played by Gravity", Submitted on 5 Dec 2008, last revised 17 Feb 2009, available at http://arxiv.org/abs/0812.1148. 